\title{
Further observations on the "Bobble-headed Doll Syndrome"
}

\author{
STEPHEN DELL
}

From the Department of Neurosurgery, Tufts-New England Medical Center, Boston, Massachusetts

SUMmARY Two patients are presented with a head-bobbling "tic" classically associated with a suprasellar cyst. The first of these showed hypothalamic, neuroendocrinological and limbic abnormalities not previously described, although with a classic arachnoidal suprasellar cyst. The second of these patients showed head-bobbling behaviour during the first years of life as a manifestation of one out of many episodes of ventricular shunt obstruction. This case is therefore the first reported whose head bobbling was observed with an acute lesion.

A relatively rare syndrome involving "truncal tremor associated with third ventricular cyst and hydrocephalus in children" was first reported by Benton et al in 1965 and has come to be known as "The Bobble-headed Doll Syndrome". ${ }^{1}$ A number of subsequent case reports have expanded and elaborated the clinical features of this syndrome. A survey by Jensen et al identified eight similar cases in addition to the nine published in the literature; to this was added another case report bringing the total to 18. ${ }^{2}$ This survey enlarged the pathologic mechanisms to include acqueductal stenosis and craniopharyngioma, in addition to the cysts of the third ventricular and suprasellar region already reported. One further third ventricular cyst has been described. ${ }^{3}$

The precise pathophysiology of this disorder is unknown, but most authors have suggested that the "tic" is related to a variety of tumours seen in conjunction with frontal lobe, corpus callosal, or basal ganglia disorders. ${ }^{4}$ All patients have presented with severe and slowly progressive hydrocephalus, which may be related to the mild but significant enlargement of the third ventricle seen on air study or CT scan in a variety of movement disorders. ${ }^{5}$ On the basis of the known somatotopic motor representation in the dorsomedial nucleus of the thalamus, the proximity of the medial aspect of this nucleus to

Address for reprint requests: Stephen Dell, MD, Department of Neurosurgery, Tufts-New England Medical Center, 171 Harrison Avenue, Boston, Massachusetts 02111, USA.

Accepted 22 May 1981 the third ventricle, and the known extrapyramidal projections of this nucleus, it has been suggested that pressure on this structure is responsible for the to-and-fro movements ("rhythmia") of the head. We describe two patients with features not previously reported. The first of these showed hypothalamic, neuroendocrinological and limbic abnormalities while the second was a sequel to acute ventricular shunt obstruction.

\section{Case 1}

This patient was an 11-year-old, right-handed female first admitted for a chronic seizure disorder. She was born at approximately $6 \frac{1}{2}$ months gestation (without maternal drug use), one week after an automobile accident which precipitated premature rupture of membranes and an eight-hour spontaneous labour. Details of delivery are unclear, but the patient's birth weight was $9 \mathrm{~kg}$, and she remained 15 days in hospital (without weight gain), with persistent hypothermia before discharge. Motor and language milestones were within normal limits. "Shaking episodes" (arm and leg extension, jerking. movements of legs, staring) began at age seven months, lasting 1-2 minutes and occurring twice daily. These persisted, and were diagnosed by her local physician as a "nervous condition" and treated with an unknown medicine. At age 7-8 years she developed seizures consist ing of tonic/clonic movements lasting 1-2 minutes, associated with loss of consciousness, head turning (to either side), postictal lethargy (for up to $40 \mathrm{~min}$ ), but without tongue biting or incontinence. These fits were variable in frequency (7-84 each week), but overall had remained unchanged in recent years. There was no evidence of myoclonus or sensory seizures. Drugs had 


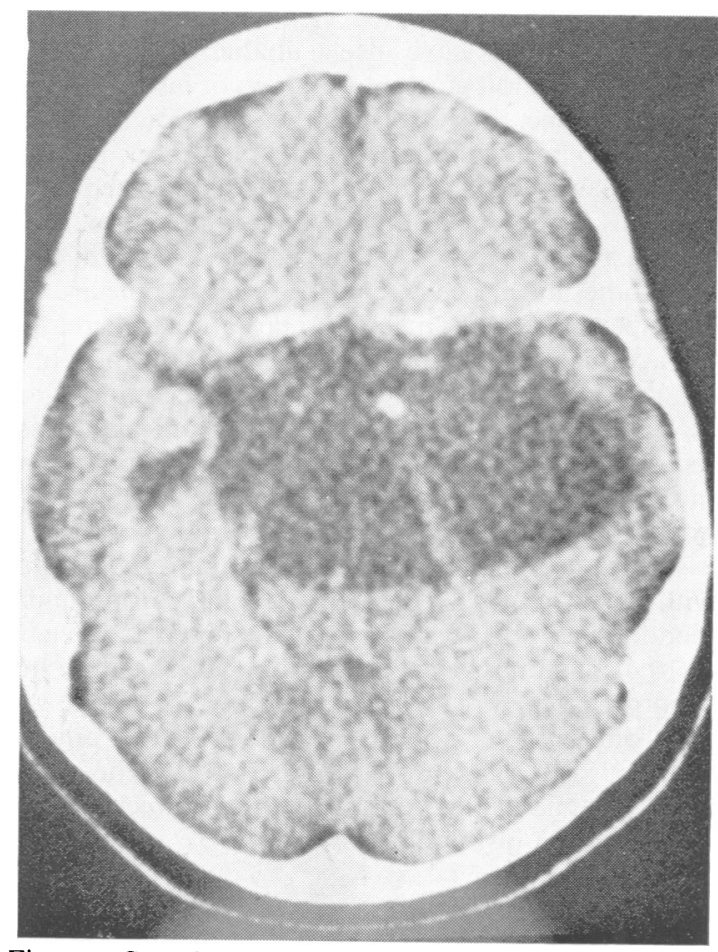

Figure Case 1: Preoperative CT.

included: diphenylhydantoin $300 \mathrm{mg}$ four times a day, primadone $500 \mathrm{mg}$ four times a day, and phenobarbital $15 \mathrm{mg}$ three times a day, but fits continued during a month-long period of observation (July, 1978). Serum levels had been adequate.

On examination the patient showed greying of her hair, with marked breast enlargement and pubic hair. She was awake and oriented but lethargic, intermittently combative, and mildly intellectually retarded. Bilateral optic atrophy mainly in the temporal field without fundal atherosclerosis, and end-gaze evoked horizontal nystagmus were the sole deficits in the cranial nerves. Motor and sensory testing were normal, but a mild dysmetria in the arms and legs was noted. Titubation was present at a rate of $1-2 /$ second, exacerbated by erect posture, and further worsened when walking. Her gait was broad-based and ataxic, with the associated head and neck "bobbling".

The diagnostic impression was of a cyst of the third ventricle, and a cyst in this region was shown by $\mathrm{CT}$ in horizontal and sagittal section (fig). The skull radiograph was normal. Hand and wrist films revealed a markedly accelerated bone age ( $17 \pm 0 \cdot 81$ years). Endocrine studies including $\mathrm{FSH} / \mathrm{LH}, \mathrm{TSH}, \mathrm{T} 3 / \mathrm{T} 4$, prolactin and diurnal cortisol levels were normal. Routine serum and cerebrospinal fluid examinations were normal. Serology for echinococcal disease was negative. Neurophthalmologic examination confirmed that the disc pallor was greater than that explicable by myopia alone. Psychological testing revealed retardation in the "educable" range (for example, WISC-R verbal IQ 47).

A right frontotemporal osteoplastic craniotomy was performed with drainage and multiple fenestration of the cyst between the right internal carotid artery optic nerve, and in the supra-chiasmatic region. Clear cerebrospinal fluid was obtained, identical to that taken preoperatively. In the prechiasmatic space, as its junction with the right optic nerve, an anomalous plexus of arteriolar and venous structures was seen. The cyst wall was shown histologically to be arachnoid membrane. The patient awoke promptly and without head movements, while her seizure frequency was much reduced $(0-2 /$ week). Although the third and lateral ventricles were reduced to 1-2+ enlargement, a persistent right frontotemporal subdural hygroma was followed with serial computed tomography. Her course was complicated by persistent spiking fevers $\left(100-104^{\circ} \mathrm{F}\right)$ showing an approximated rhythm. On two occasions, right subdural drains were placed for evaluation and drainage of possible empyema, although none were found.

After multiple negative cultures, with several empiric courses of antibiotics (including oxacillin, cloxacillin, gentamycin, chloramphenicol, vancomycin, cephalothin and bactrim), her fever spontaneously resolved. The patient was discharged to return home (Jamaica) with the neurologic improvements mentioned, but still on triple anticonvulsant therapy.

\section{Case 2}

The child was the product of a normal gestation and delivery, complicated only by lack of pre-natal care, and with Apgar scores of 8 and 9 at 1 and 5 minutes respectively. A $4.5 \times 5.0 \mathrm{~cm}$ meningomyelocele was noted at birth extending from the L2-3 level to the sacrum. The head was small $(31.5 \mathrm{~cm}$ circumference), there was excellent function in the quadriceps and Achilles groups, with good hip, knee and ankle movement, and pin sensation was preserved on the plantar surfaces bilaterally. Anal sphincter tone was diminished and there was no apparent sensation in the perineum. Associated anomalies included bilateral hydrouretor with a markedly dilated bladder. Closure of this partially epithelialised but leaking meningomyelocele was performed on the first day of life and by the eighth day, increasing head circumference necessitated the placement of a ventriculoperitoneal shunt. Three further shunt revisions were needed during the early months for recurrent obstructions.

At age 5 months, intermittent bobbling movements of the head were noted for the first time. Examination revealed a head circumference of $42 \mathrm{~cm}$ and a $3 \times 3 \mathrm{~cm}$ intermittently tense anterior fontanelle. There was moderate limitation of upward and lateral gaze, with Collier's sign (lid retraction) being present. Vertical bobbling of the head at a frequency of $2 / \mathrm{s}$ was noted for extended periods unaccompanied by nystagmus or truncal movement. This could be elicited only in the seated position and was abolished by the supine posture. Head control at this time was poor. The left leg was externally rotated, deep tendon reflexes were hypoactive, and ankle jerks were absent. The remainder of the neurological examination was unchanged from that previously noted. The patient's increasing head circumference, full fonta- 
nelle, eye findings and poor filling of the shunt on manual examination were believed to imply shunt malfunction but a CT scan revealed ventricles which, although larger than normal, were less dilated than at any prior CT examination. The head bobbling became more frequent. Within a month, irritability and a persistently bulging anterior fontanelle prompted revision of the shunt once again. Although further episodes of shunt malfunction necessitated shunt revision on three occasions in the ensuing eight months, no further head bobbling could be observed. Since that time (November 1978), the patient has been free from either head bobbling or shunt obstruction.

\section{Discussion}

Case reports of the bobble-headed doll syndrome have almost always shown an association with cysts in or about the third ventricle. ${ }^{6-9}$ Pathology in this region is, however, rarely associated with the bobbleheaded doll syndrome, there being mainly a midline syndrome of increased intracranial pressure without lateralising signs, or a hypothalamo-pituitary syndrome of direct compression. 1011 It is likely that additions to the small number of reported cases will present aspects of the syndrome not hitherto noted. It is for this reason that these cases with certain unique features are described.

In the first patient's course, a thermoregulatory abnormality is strongly suggested by her neonatal history as well as by the persistent postoperative febrile course, extending over months beyond hospital discharge, without evidence for an inflammatory or infective process. Menses commenced in this patient at age $9 \mathrm{yr}$, and by age $11 \mathrm{yr}$ secondary sexual characteristics were well-developed. Of equal note was the remarkable thinning and greying of hair, already evident in the pubertal period. Her attacks of rage could be severe, and included assaults upon other patients. These attacks appeared to be unrelated to seizures, although simultaneous electroencephalography was not performed. In view of the compression by the arachnoidal cyst in the region of the third ventricle, correlation with hypothalamic, neuroendocrinological and limbic dysfunctions are reasonable.

The second patient demonstrated her head-bobbling at age 5 months, which would appear to be the youngest such case in the literature. However, onset of the syndrome in the first 2 years of life is not uncommon, and the average age at time of diagnosis is 7 years. $^{2}$ Of greater interest is this child's presentation with acute obstruction of a ventriculoperitoneal shunt which has never hitherto been reported to produce the syndrome. (For this reason simple hydrocephalus or elevated intracranial pressure is insufficient explanation of the Bobble- headed Doll Syndrome.)

Most authors have been unable to suggest a coherent, pathological physiology or anatomy for the syndrome. In its original description, the distinctive tremor, inhibited by intended movement or volition, was remarked upon and contrasted with familial tremor, extrapyramidal dysfunction, and habit spasm, or tic. Other concomitants of the syndrome have been remarked upon but are as non-specific (mild mental retardation, increased intracranial pressure, optic atrophy, strabismus, and long-tract signs). ${ }^{24}$ The results of decompression have been variable, even when mechanically satisfactory, and their correlation with antecedent intracranial pressure appears almost random. Of greatest interest has been the rather constant association of a third ventricular or suprasellar cyst with this syndrome. The theory of Russo and Kindt, identifying pressure upon the dorsomedial nucleus of the thalamus as the anatomical site for the syndrome, has already been mentioned.12 While the syndrome would appear to be classified among those of "resting tremor", and hence identified with basal ganglia structures, further anatomical localisation is not justifiable. ${ }^{13} 14$

\section{References}

${ }^{1}$ Benton JW, Nellhaus G, Huttenlocher PR, Ojemann RG, Dodge PR. The Bobble-head Doll Syndrome. Neurology (Minneap) 1966;16:725-9.

2 Jensen HP, Pendl G, Goerke W. The Head-bobbing in a patient with a cyst of the third ventricle. Child's Brain 1978;4:235-41.

3 Patriquin HB. The Bobble-head Doll Syndrome: A curable entity. Radiology 1973;107:171-2.

${ }^{4}$ Nellhaus G. The Bobble-head Doll Syndrome: "Tic" with a neuropathologic basis. Pediatrics 1967;40: 250-3.

5 Nelson SR, Andy OJ, Foshee DP. Third ventricle size in patients with movement disorders. Confin Neurol 1964;24:308-13.

${ }^{6}$ Ferry PC, Feldman V, Kerber C. The Bobble-head Syndrome, due to a basal arachnoid cyst (abs). Neurology (Minneap) 1974;24:394.

${ }^{7}$ Mayher WE, Gindin RA. Head-bobbing associated with third ventricular cyst. Arch Neurol 1970;23: 274-7.

8 Obenchain TG, Becker DP. Head-bobbing associated with a cyst of the third ventricle. J Neurosurg 1972; 37:457-9.

9 Sakata K, Yamada H, Yamamoto M. Bobble-head Doll Syndrome. Brain and Neurol (Tokyo) 1971 ;23: 693-700.

${ }^{10}$ Raimondi AJ, Shimoji T, Gutierrez FA. Suprasellar cysts: Surgical treatment and results. Child's Brain 1980;7:57-72. 
"Sansregret A, Ledoux R, Duplantis R, Lamoureux C, Chapdelaine A, Leblanc P. Suprasellar subarachnoid cysts: radioclinical features. $A J R$ 1969;105: 291-7.

12 Russo RH, Kindt GW. A neuroanatomical basis for the Bobble-head Doll Syndrome. J Neurol 1974;41 : 720-3.

${ }^{13}$ Carpenter MB. Human neuroanatomy. The Basal

Ganglia. Baltimore: Williams and Wilkins, 1976: 496-520.

${ }^{14}$ Cooper IS, Bravo GJ. Anterior choroidal artery occlusion, chemopallidectomy and chemothalamectomy in Parkinsonism. In: Field WS, ed. Pathogenesis and Treatment of Parkinsonism. Springfield: Charles C Thomas, 1958:325-52. 